\title{
Implementación de un Control Predictivo Basado en Modelo Aplicado a un Sistema de Control de Caudal de Agua Didáctico
}

\author{
Edwin H. Lopera ${ }^{1}$ \\ Diego A. Mejía ${ }^{2}$
}

\section{Resumen}

El controlador proporcional integral derivativo -PID- se ha convertido en la herramienta de regulación de variables de proceso más utilizada, y por ello se ha aplicado de forma indiscriminada sobre sistemas dinámicos lineales y no lineales, generando problemas de control en el sector industrial, produciéndose pérdida de eficiencia en la productividad y disminución de la calidad de los productos manufacturados. Dentro de las técnicas de control moderno que han surgido para responder en aplicaciones en las cuales el controlador PID no ha operado satisfactoriamente, está el control predictivo basado en modelo -CPM-. Este algoritmo de control se ha caracterizado por su gran capacidad de respuesta, sin embargo, su expansión en el sector industrial ha sido lenta, ya que ha sido comercializado para aplicaciones particulares. Este artículo presenta resultados experimentales de la aplicación de un CPM sobre la regulación de caudal de agua en un sistema didáctico, utilizando equipos de control e instrumentación comerciales, describiéndose en el proceso la formulación del algoritmo de control, las intervenciones técnicas requeridas para la ejecución de las pruebas experimentales necesarias para obtener el modelo matemático de la planta y aplicar finalmente la técnica de control propuesta. Los resultados obtenidos evidencian las grandes

1 Facultad de Ingeniería, Ingeniería Electromecánica, INSTITUTO TECNOLÓGICO METROPOLITANO, edwinlopera@itm.edu.co

2 Facultad de Ingeniería, Ingeniería Eléctrica, Universidad de Antioquia, diegomej@udea.edu.co

Fecha de recepción: 14 de Agosto de 2010

Fecha de aceptación: 20 de Octubre de 2010 
posibilidades de aplicar eficientemente esta técnica de control en la regulación de variables de sistemas dinámicos generales.

\section{Palabras clave}

Control predictivo, control de caudal, identificación de sistemas, optimización matemática.

\section{Abstract}

The proportional-integral-derivative controller -PID- has become the most used tool of regulation of process variables, and so it has been applied indiscriminately on linear and nonlinear dynamic systems, resulting in control problems in the industry, occurring loss of efficiency in productivity and decreased quality of manufactured products. Among the modern control techniques that have emerged to respond to those applications in which the PID controller has not operated satisfactorily, there is the model based predictive control -MPC-. This control algorithm has been characterized by its great capacity to respond, however, its expansion in the industrial sector has been slow, as it has been commercialized for specific applications. This paper presents an application of MPC on the regulation of water flow in a laboratory system using commercial control equipment and instrumentation, describing the formulation process control algorithm, the technical assistance required for the execution of experimental tests necessary to obtain the mathematical model of the plant and finally implementing the proposed control technique. The results evidence the great possibilities of applying effectively this control technique in variable regulation of general dynamic systems.

\section{Keywords}

Flow control, mathematical optimization, model predictive control, system identification. 


\section{INTRODUCCIÓN}

El controlador proporcional integral derivativo -PID- ha sido el algoritmo más utilizado durante las últimas décadas para resolver los problemas de control de las variables de los procesos industriales; se estima que el $95 \%$ de los controladores implementados son de este tipo. Este controlador se mantiene como una herramienta de regulación de gran aceptación a nivel industrial fundamentalmente por sus antecedentes de éxito en gran variedad de aplicaciones, por su amplia disponibilidad en el mercado y por la simplicidad de su uso e implementación (O'dwyer, 2006). Además, se ha demostrado que la estructura del control digital aplicada a sistemas de orden superior, no ha sido realmente capaz de desplazarlo, ya que sólo en los casos en que la dinámica de los procesos es demasiado compleja los ingenieros de control consideran el uso de técnicas y algoritmos más avanzados y más sofisticados (Qin \& Badgwell, 2003).

Aunque prácticamente todos los procesos industriales presentan un comportamiento dinámico no lineal en alguna parte de su zona de operación, en su gran mayoría todavía se están controlando con las estructuras del regulador PID clásicas. Desafortunadamente, estos reguladores solamente pueden ser sintonizados con buenos resultados alrededor de un punto de operación definido, y para obtener respuestas apropiadas sobre todo el rango de operación de las variables, se requiere una adaptación en línea de sus parámetros, lo cual no se está logrando, pues se ha documentado que cerca del $80 \%$ de los controladores industriales se encuentran mal sintonizados y que aproximadamente el $30 \%$ de ellos operan gran parte del tiempo en modo manual (O'dwyer, 2006).

Una de las estrategias de control avanzado que se ha estudiado con profundidad para suplir estas falencias en la regulación de las variables de los procesos, es el Control Predictivo basado en Modelo -CPM-. Esta estrategia es considerada como la única metodología de control avanzado que ha sido realmente exitosa en aplicaciones prácticas, influenciando notablemente las directrices del desarrollo de algunos sistemas de control industriales y las 
investigaciones en el campo del saber de la teoría del control (Fernández \& Bordons, 2004b; Lawrynczuk, 2007).

Las primeras aplicaciones del CPM se presentaron en los años setenta, cuando el ingeniero Jacques Richalet propuso un algoritmo denominado Control Heurístico Predictivo de Modelo (Model Predictive Heuristic Control - MPHC), el cual pretendía resolver problemas bastante complejos en donde el controlador convencional PID no había respondido apropiadamente, pero que estuviese basado en conceptos intuitivos $y$ ofreciera fácil sintonización.

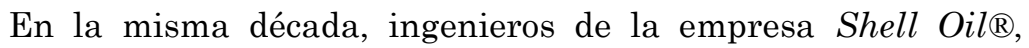
aplicaron un algoritmo de control multivariable sin restricciones que llamaron Control Matricial Dinámico (Dynamic Matrix Control- DMC), el cual más adelante fue modificado para manejar no linealidades y restricciones de los procesos. El objetivo del controlador DMC era conducir la salida de la planta tan cerca de la consigna como fuera posible, con un término de penalidad sobre los cambios de las variables manipuladas. Esto permitió que durante el control de un proceso se obtuvieran variaciones más pequeñas en las señales de entrada y que las respuestas de las variables controladas fueran menos abruptas. El DMC fue el producto de control predictivo más aplicado durante estos primeros años.

En los años ochenta, ingenieros de la misma empresa presentaron una sustancial mejora a los algoritmos previos, permitiendo que los usuarios pudieran definir de forma explícita las restricciones de las señales de entrada y de salida. El algoritmo QDMC (Quadratic Dynamic Matrix Control), diseñado por ellos, puede ser considerado como una segunda generación de la tecnología CPM, ya que permitía una mayor rentabilidad a través de la optimización en línea, ofreciendo igualmente una transición suave entre diferentes puntos de operación cercanos a las restricciones de las variables.

La tercera generación de algoritmos CPM surgió principalmente por la necesidad de considerar la solución de los problemas de control a partir de varias funciones objetivo y tomar decisiones sobre problemas mal condicionados. Es así como varios fabricantes, a finales de los años ochenta, introdujeron al mercado 
varios algoritmos, tales como: IDCOM-M (Identification and Command), HIECON (Hierarchical Constraint Control), SMCA (Setpoint Multivariable Control Architecture) y SMOC (Shell Multivariable Optimizing Controller).

En la década de los noventa, la cuarta generación de algoritmos CPM surge como resultado de la integración de varias de las empresas que venían ofreciendo estos productos. La competencia entre los vendedores de la tecnología CPM ha permitido que se establezcan en la actualidad básicamente dos algoritmos bastante funcionales de aplicación industrial. Esta última generación de controladores industriales, fundamentada básicamente en los productos DMC-plus (Dynamic Matrix Control Package - DMC+) y RMPCT (Robust Model Predictive Control Technology), se caracteriza por utilizar interfaces gráficas de usuario; disponer de niveles de optimización múltiples para priorizar los objetivos del controlador; incluir funciones objetivo económicas; considerar directamente la incertidumbre del modelo; y disponer de tecnología de identificación mejorada; entre otras ventajas (Maciejowski, 2004).

El CPM ha sido considerando un referente industrial para el control de procesos multivariables con restricciones, por lo que se constituye hoy en día en una de las herramientas más poderosas para el control de procesos tecnológicos industriales, pues su estructura interna, sustentada en un proceso de optimización, permite predecir el desempeño futuro de las variables de la planta y sus señales de control, basándose fundamentalmente en el conocimiento de las variables que caracterizan el proceso (Rodríguez \& León, 2002).

Esta metodología no se refiere a una estrategia de regulación particular, sino más bien a un conjunto de algoritmos de control que hacen uso explícito de un modelo de proceso para obtener la señal de control, optimizando una función objetivo en tiempo real. Estos métodos de control conllevan a la implementación de reguladores que tienen básicamente la misma estructura y los mismos elementos (Cao \& Lin, 2005; Fernández \& Bordons, 2004a). Los diversos algoritmos del CPM difieren entre si principalmente en el modelo utilizado para representar el proceso 
que se desea controlar y en la función objetivo que va a ser minimizada (Maciejowski, 2004).

Las ventajas más representativas de esta tecnología se obtienen al considerar el modelo del proceso y las restricciones de las variables en su formulación, lo cual permite que el controlador trabaje con una réplica exacta o muy aproximada de la dinámica del sistema real, obteniéndose de esta forma una mejor calidad en el desempeño del control de las variables (Jalili \& Araabi, 2004). En cuanto a las desventajas de este algoritmo, se ha evidenciado que el tiempo requerido para su ejecución por parte del equipo de procesamiento puede limitar su aplicación sobre sistemas de dinámicas rápidas, ya que eventualmente no se puede obtener una señal de control óptima con la velocidad que requiere el sistema. Por otro lado, también se ha reconocido que en muchos casos puede ser difícil obtener el modelo teórico o experimental de la planta, lo cual es el insumo esencial para el planteamiento del problema de optimización. Estas son las principales causas que finalmente han llevado a que su expansión en el control de procesos industriales haya sido bastante lenta (Hirano et al., 2001).

Considerando un sistema dinámico como el esquematizado en la Fig. 1, el principal objetivo del controlador es mantener la variable de salida $(\bar{y})$ tan cerca como sea posible de la señal de referencia $(\boldsymbol{r})$, ajustando la variable manipulada $(\boldsymbol{u})$. El bloque denominado CPM representa el Controlador Predictivo basado en Modelo usado para alcanzar los objetivos de control.

Además de la variable manipulada, pueden existir señales de perturbación medibles $(\boldsymbol{v})$, y señales de perturbación no medibles (d), las cuales representan aquellos fenómenos que generan cambios inesperados en el comportamiento de la variable de salida. Dado que las perturbaciones no medibles siempre estarán presentes, en el diagrama de bloques se observa que ésta es una señal independiente que no puede ser afectada directamente por el controlador. Esta señal representa todos los eventos impredecibles que afectan el desempeño de la planta.

Por su parte, la señal de ruido (z), está asociada a ruidos eléctricos, errores de muestreo, problemas de calibración de los 
equipos y otros fenómenos que pueden afectar la precisión y exactitud de las mediciones (Bemporad et al., 2008).

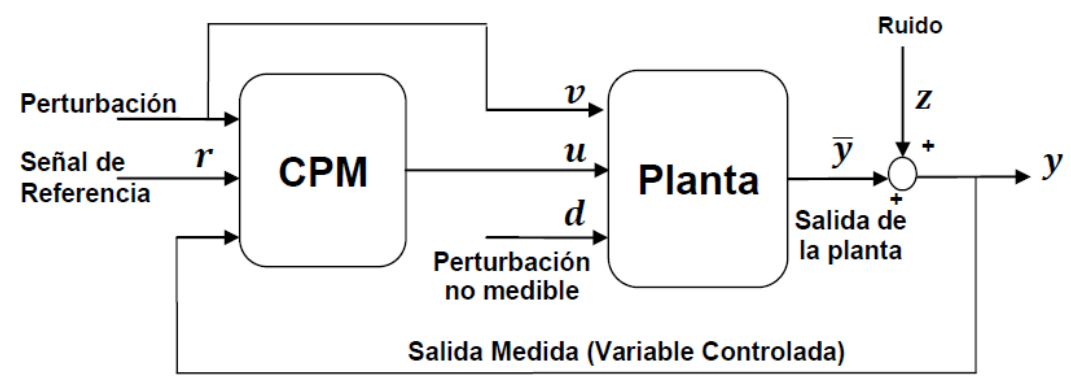

Fig. 1. Diagrama de bloques de un CPM

Como se observa en el diagrama, el controlador recibe directamente las perturbaciones medibles por lo cual puede compensarlas inmediatamente antes de que sus efectos se reflejen sobre la variable de salida (y). Esta característica es la que se conoce como control prealimentado. Se evidencia entonces que el CPM siempre permite aplicar la compensación realimentada para las perturbaciones no medibles y la compensación prealimentada para cualquier perturbación medible.

El CPM genera un control en tiempo discreto y actúa sobre un intervalo de tiempo específico denominado Intervalo de Control. En la Fig. 2, el valor entero $\mathrm{k}$ representa un instante de tiempo presente, y se considera que los valores de la última señal de salida medida $\left(\boldsymbol{y}_{\boldsymbol{k}}\right)$ y las medidas previas $\left(\boldsymbol{y}_{\boldsymbol{k}-\mathbf{1}}, \boldsymbol{y}_{\boldsymbol{k}-2}, \ldots\right)$ son conocidas.

En la misma Fig. 2 se muestra el comportamiento previo de la señal de control. Para el cálculo del próximo movimiento, el CPM opera en dos fases: estimación y optimización. En la fase de estimación, el controlador utiliza todos los cambios previos, los valores actuales de las variables conocidas y el modelo de la planta para predecir el desempeño de la variable de salida, mientras que en la fase de optimización, los valores de la señal de referencia, las perturbaciones medibles y las restricciones, se especifican para un Horizonte de Predicción o instantes de muestreo posteriores, y con esta información el controlador calcula varios movimientos futuros 
de la variable manipulada sobre un Horizonte de Control, los cuales corresponden a la solución de un problema de optimización con restricciones.

Terminado este proceso, el controlador aplica la señal de control restringida $\left(\boldsymbol{u}_{\boldsymbol{k}}\right)$ a la planta, la cual opera con esta entrada hasta el próximo Intervalo de Control, donde el controlador toma nuevas mediciones para repetir los procesos de estimación y optimización.
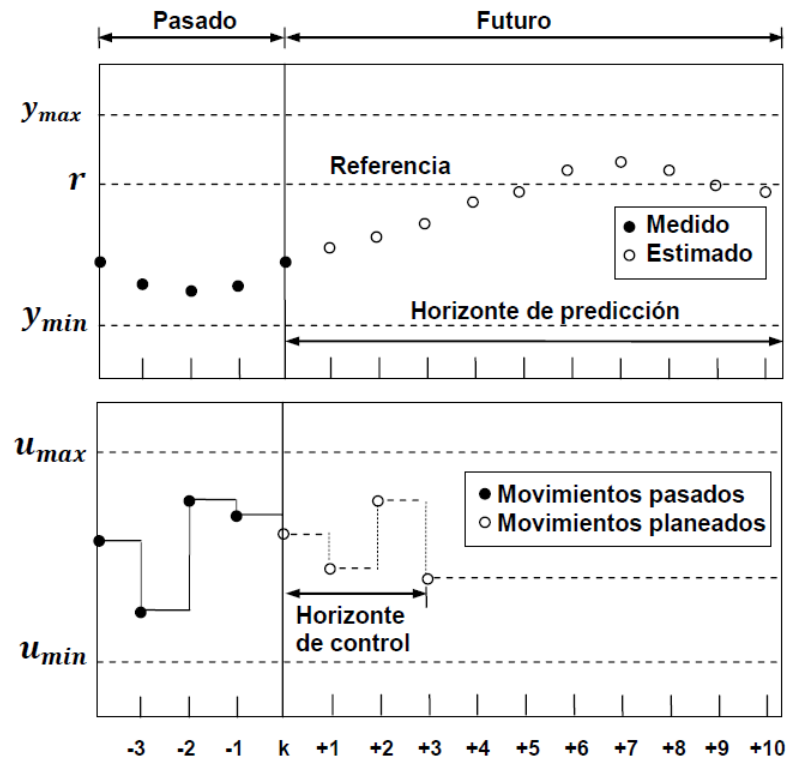

Fig. 2. Operación de un CPM

\section{METODOLOGÍA}

Para mostrar las implicaciones en la implementación de un CPM lineal en un proceso automatizado de uso frecuente en el ámbito industrial, como lo es el control de caudal, a continuación se describen los aspectos técnicos y teóricos que se tienen en cuenta para la aplicación de esta estrategia de control sobre un 
sistema dinámico didáctico que inicialmente utiliza instrumentación industrial convencional.

\subsection{Materiales y Métodos}

Para llevar a cabo las pruebas experimentales con el CPM se utiliza un sistema dinámico didáctico que permite realizar diversos estudios sobre la medición y el control de caudal y de nivel de agua. Este sistema está ubicado en el Laboratorio de Fluidos del INSTITUTO TECNOLÓGICO METROPOLITANO y desde hace varios años se viene interviniendo para incorporarle nuevas tecnologías que incrementen las posibilidades de aplicación de estrategias de control convencionales y avanzadas, exclusivamente para fines académicos. Los nuevos equipos que se han incorporado mejoran la instrumentación del sistema y posibilitan la adaptación de controladores de propósitos industriales que permiten realizar prácticas académicas y de investigación sobre las variables físicas mencionadas (Ramírez \& Londoño, 2007).

Para aplicar estrategias de control avanzadas como el CPM sobre este sistema se hace necesario incorporar nuevos elementos de hardware y de software, sin que ello menoscabe las posibilidades de aplicación que en un principio ofrece el sistema. Por lo tanto, la aplicación del CPM obliga a reestructurar su configuración inicial y exige la integración de dispositivos importantes tales como tarjetas de adquisición de datos y convertidores de señales, que permitan aplicar la estrategia de control directamente desde un equipo de cómputo. En la Fig. 3 se esquematiza el diagrama de instrumentación inicial del sistema.

Tal como se muestra en la Fig. 3, los componentes generales del sistema dinámico para el control de caudal y de nivel corresponden a: un tanque cilíndrico; un tanque construido en lámina metálica; dos transmisores e indicadores electrónicos para la medición de nivel de cada tanque; dos transmisores electrónicos y sus respectivos componentes complementarios, que miden el caudal de líquido que se transporta desde el tanque metálico hasta el tanque de acrílico, el cual puede ser regulado usando un variador de frecuencia, que actúa sobre una bomba centrífuga 
trifásica, o a través de una válvula neumática proporcional; una bomba centrífuga monofásica para transportar agua hacia el tanque metálico; detectores de nivel y de caudal que pueden programarse para evitar el desbordamiento del líquido de los tanques y la operación en vacío de las bombas centrífugas; dos electroválvulas para controlar el ingreso de fluido a cada uno de los tanques; un controlador universal; un controlador lógico programable y un equipo de cómputo.

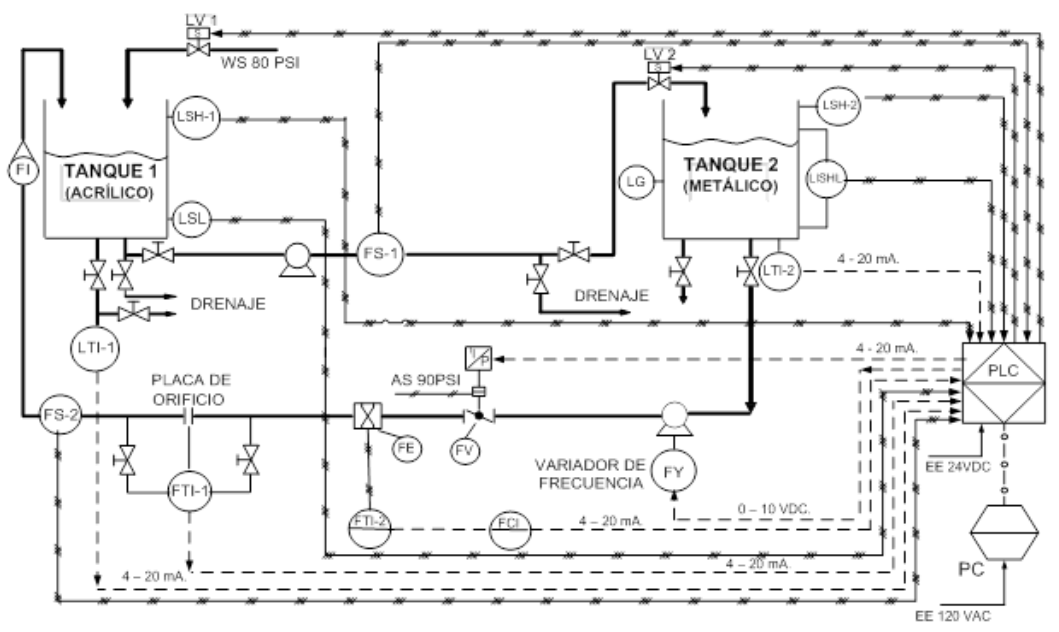

Fig. 3. Diagrama de instrumentación inicial del sistema dinámico

El primer paso para la implementación del controlador consiste en obtener el modelo matemático de la planta. En este caso, se realizan diversas pruebas experimentales para definir un modelo matemático aproximado que relacione el comportamiento del caudal de fluido desde el tanque 1 hacia el tanque 2 , con respecto a la variación de frecuencia y voltaje de la bomba trifásica, en una zona de operación lineal. Como restricciones del sistema se consideran los límites de operación de la velocidad de la bomba trifásica y el caudal sobre la tubería. Cabe anotar que por seguridad en todo momento se restringirá la tasa de cambio de velocidad del variador de frecuencia para disminuir riesgos 
durante cambios bruscos de los valores de referencia de las variables.

El modelo de la planta se puede aproximar al de un sistema lineal invariante en el tiempo, el cual se describe por (1):

$$
\begin{aligned}
& x(k+1)=A x(k)+B_{u} u(k)+B_{v} v(k)+B_{d} d(k) \\
& y_{m}(k)=C_{m} x(k)+D_{v m} v(k)+D_{d m} d(k) \\
& y_{u}(k)=C_{u} x(k)+D_{v u} v(k)+D_{d u} d(k)+D_{u u} u(k)
\end{aligned}
$$

donde, $x(k)$ es el vector de estados de la planta, $u(k)$ es vector de variables manipuladas, $v(k)$ es vector de perturbaciones medibles, $d(k)$ es vector de perturbaciones no medibles, $y_{m}(k)$ es el vector de las salidas medibles, $\mathrm{y} y_{u}(k)$ es el vector de salidas no medibles. $y(k)$ es el vector de salidas que recoge $y_{m}(k)$ y $y_{u}(k)$. Considerando que el valor estimado de $x(k)$ está disponible en el tiempo $k$, la acción de control se obtiene resolviendo el problema de optimización presentado en (2). Minimizar:

$$
\begin{aligned}
& \Delta u(k \mid k), \ldots, \Delta u(m-1+k \mid k), \\
& \varepsilon\left\{\left.\sum_{i=0}^{p-1}\left(\sum_{j=1}^{n_{y}} \mid w_{i+1, j}^{y}\left(y_{j}(k+i+1) \mid k\right)-r_{j}(k+i+1)\right)\right|^{2}\right. \\
& +\sum_{j=1}^{n_{u}}\left|w_{i, j}^{u}\left(u_{j}(k+i \mid k)-u_{j_{\text {OBJETIVO }}}(k+i)\right)\right|^{2} \\
& \left.\left.+\sum_{j=1}^{n_{u}}\left|w_{i, j}^{\Delta u} \Delta u_{j}(k+i \mid k)\right|^{2}\right)+\rho_{\varepsilon} \varepsilon^{2}\right\}
\end{aligned}
$$

donde el subíndice " $j$ " denota el $j$-ésimo componente del vector, "( $k+i \mid k)$ " denota el valor predicho para el tiempo $k+i$ basado en la información disponible en el tiempo $k ; r(k)$ es la muestra actual de la señal de referencia; $n_{y}$ corresponde al 
[120] Implementación de un Control Predictivo Basado en Modelo Aplicado a un Sistema de Control de Caudal de Agua Didáctico

Horizonte de Predicción y $n_{u}$ al Horizonte de Control. Las restricciones de este problema de optimización se plantean en (3). Sujeto a:

$$
\begin{aligned}
& y_{j \min }(i)-\varepsilon V_{j \min }^{y}(i) \leq y_{j}(k+i \mid k) \leq y_{j \max }(i)+\varepsilon V_{j \max }^{y}(i) \\
& u_{j \min }(i)-\varepsilon V_{j \min }^{u}(i) \leq u_{j}(k+i \mid k) \leq u_{j \max }(i)+\varepsilon V_{j \max }^{u}(i) \\
& \Delta u_{j \min }(i)-\varepsilon V_{j \min }^{\Delta u}(i) \leq \Delta u_{j}(k+i \mid k) \leq \Delta u_{j \max }(i)+\varepsilon V_{j \max }^{\Delta u}(i), \\
& i=0, \ldots, p-1 \\
& \Delta u(k+h \mid k)=0, \quad h=m, \ldots, p-1 \\
& \varepsilon \geq 0
\end{aligned}
$$

con respecto a la secuencia de incrementos de la entrada y el factor $\varepsilon$, ajustando $u(k)=u(k-1)+\Delta u(k \mid k)^{*}$, donde $\Delta u(k \mid k)^{*}$ es el primer elemento de la secuencia óptima. $w_{i, j}^{\Delta u}, w_{i, j}^{u}, w_{i, j}^{y}$, son los pesos para la variable correspondiente, mientras que $u_{\text {jmin }}, u_{j \max }, \Delta u_{j \min }, \Delta u_{j \max }, y_{\text {jmin }}, y_{\text {jmax }}$ indican los límites mínimo y máximo de cada variable. El peso $\rho_{\varepsilon}$ y el factor $\varepsilon$ penalizan la violación de las restricciones. Los vectores $V_{\text {min }}^{u}, V_{\text {max }}^{u}, V_{\text {min }}^{\Delta u}, V_{\text {max }}^{\Delta u}, V_{\text {min }}^{y}, V_{\text {max }}^{y} \quad$ se ocupan de relajar las correspondientes restricciones. El vector $u_{j_{\text {OBJETIVO }}}(k+i)$ es una referencia para el vector de entradas (Bemporad et al, 2008).

\section{RESULTADOS Y DISCUSIÓN}

\subsection{Ajuste Experimental}

En la Fig. 4 se muestra la nueva distribución de los equipos después de las intervenciones ejecutadas sobre el sistema de pruebas, y en la Fig. 5 se evidencia el estado en quedan los tableros de control, los cuales permiten desarrollar pruebas experimentales con controladores industriales convencionales, tales como un controlador lógico programable o un controlador PID 
universal; o para la implementación de controladores digitales avanzados, tales como el CPM, usando en este caso un equipo de procesamiento externo y las tarjetas de adquisición instaladas. Todo esto aplicable al control de caudal y de nivel.

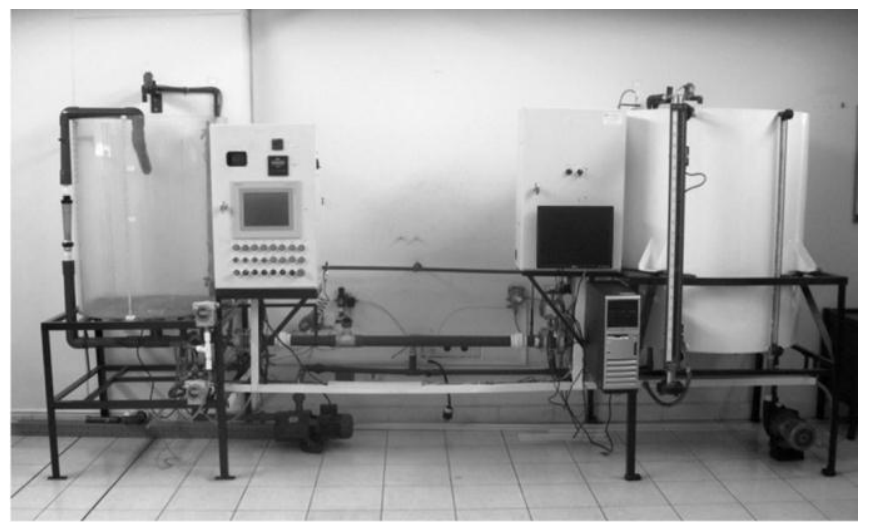

Fig. 4. Distribución de los equipos después de las intervenciones ejecutadas

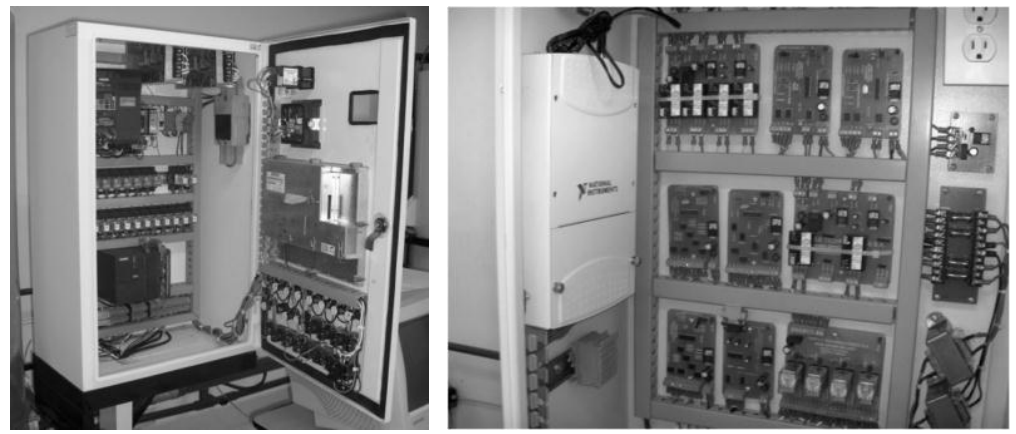

Fig. 5. Reconfiguración e instalación de los equipos de control del sistema

En resumen, se instaló un nuevo tablero de control con tarjetas de adquisición de datos, con un equipo de cómputo actualizado, y se reconfiguraron los equipos instalados y todo el cableado en el tablero original. Ambos tableros de control quedaron integrados de tal manera que se puede utilizar cualquiera de los controladores disponibles de forma independiente. 


\subsection{Esquema Propuesto}

Considerando la instrumentación requerida para la implementación del algoritmo de control sobre el sistema, en la Fig. 6 se discriminan los equipos utilizados de forma directa para realizar las pruebas experimentales, los cuales inicialmente permiten definir un modelo matemático lineal para el caudal y, posteriormente, aplicar el CPM lineal. Cabe anotar que existen otras variables e instrumentos del sistema que intervienen de forma indirecta en la aplicación de los algoritmos, los cuales están relacionados con la operación segura del sistema, tal como ocurre en cualquier sistema automático real. Estos instrumentos permiten, entre otras acciones, evitar el rebose de los tanques, la operación en vacío de las bombas centrífugas o la intervención de la válvula neumática en las pruebas, y controlar el ingreso del líquido a los tanques de acuerdo a las necesidades.

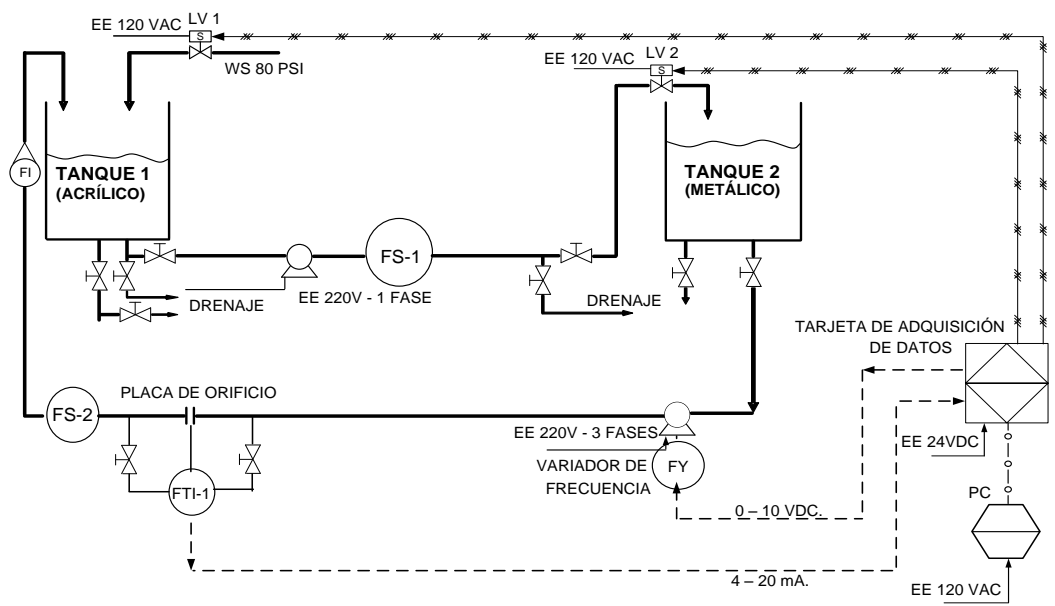

Fig. 6. Equipos utilizados para la identificación del sistema y la aplicación del CPM

Para determinar las posibles zonas de operación lineal del sistema se aplica una señal de entrada correspondiente a la frecuencia eléctrica aplicada por el variador de frecuencia. Esta señal está formada por una serie de pequeños escalones sucesivos, 
de forma ascendente y descendente sobre todo el rango de operación de las dos variables involucradas, de tal forma que frente a cada uno de ellos se pueda evidenciar la respuesta transitoria del caudal hasta su estabilización. El resultado de esta prueba se muestra en la Fig. 7, y constituye la respuesta en lazo abierto del proceso, usada normalmente para la identificación de sistemas dinámicos lineales.

En la Fig. 7 se observa, entre otros factores, que se aplicaron escalones de $5 \mathrm{~Hz}$ de amplitud con una duración de 7 segundos cada uno, y que el caudal máximo del sistema para la frecuencia nominal de la bomba es de $39 \mathrm{gal} / \mathrm{min}$.

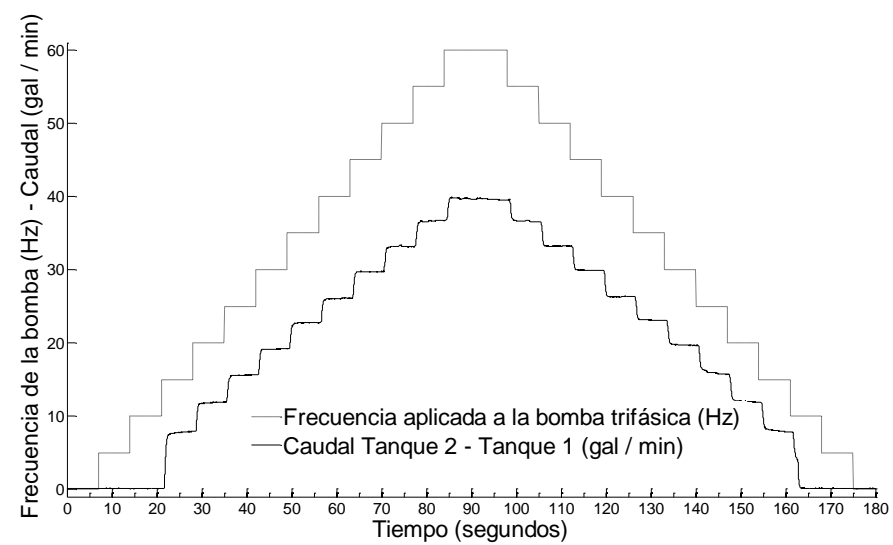

Fig. 7. Respuesta del caudal frente a variaciones en la frecuencia de la bomba

Para evitar problemas con la respuesta del variador de frecuencia y la bomba trifásica frente a estos cambios bruscos de sus variables electromecánicas por la aplicación de estas señales de excitación, fue necesario programar pequeñas rampas de aceleración y desaceleración de 0,1 segundos desde el mismo variador, lo cual también fue tenido en cuenta como una restricción del sistema en la implementación del CPM. Con la tarjeta de adquisición de datos utilizada se adquirieron señales con un periodo de muestreo de $1 \mathrm{~ms}$.

Evaluando los valores de estabilización de la variable caudal frente a cada una de las señales tipo escalón aplicadas, se obtuvo 
la curva estática o de linealidad mostrada en la Fig. 8. En esta gráfica se observa que la planta presenta una zona de operación aproximadamente lineal entre $10 \mathrm{gal} / \mathrm{min}$ y $35 \mathrm{gal} / \mathrm{min}$. Sin embargo, se evidencia una leve histéresis en la zona de operación intermedia, aproximadamente entre $15 \mathrm{gal} / \mathrm{min}$ y $30 \mathrm{gal} / \mathrm{min}$. Por debajo de los $10 \mathrm{~Hz}$ no se genera un caudal apreciable en la tubería, debido a la cabeza de presión que debe vencer la bomba para lograr la descarga de agua por la parte superior del tanque de acrílico (ver Fig. 4). Esta situación es la que genera la mayor no linealidad de la planta para el control del caudal.

Finalmente, realizada una corta prueba por encima de los 60 $\mathrm{Hz}$ para no maltratar la bomba trifásica, se notó que a los $70 \mathrm{~Hz}$ comienzan a aparecer signos apreciables de saturación de la señal de caudal, lo cual se puede apreciar levemente en la Fig. 8, por encima de los $35 \mathrm{gal} / \mathrm{min}$.

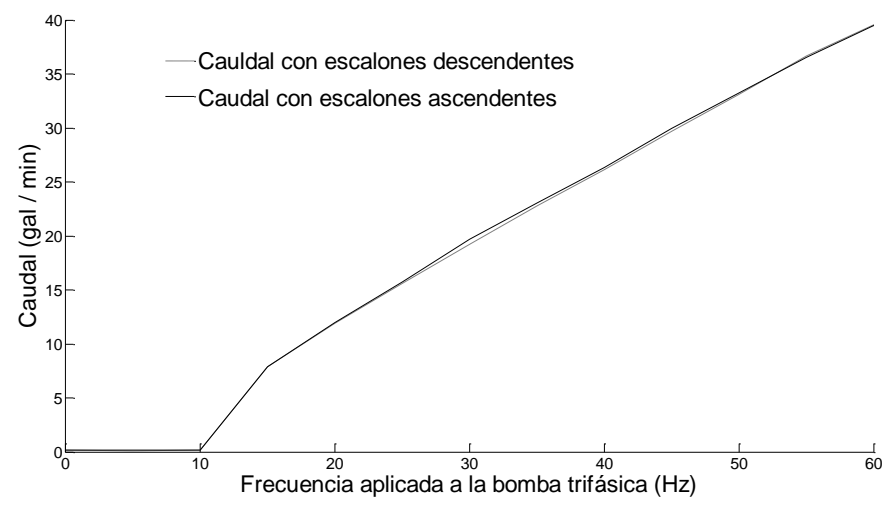

Fig. 8. Curva estática o de linealidad del sistema

Evaluando con mayor detalle la zona claramente no lineal de esta planta, es decir, por debajo de los $10 \mathrm{gal} / \mathrm{min}$, se aplicaron pequeños escalones de $1 \mathrm{~Hz}$, desde $0 \mathrm{~Hz}$ hasta $15 \mathrm{~Hz}$, y se observó que se requieren al menos $7 \mathrm{~Hz}$ para producir un caudal mínimo visible, que el transmisor en esas condiciones no es capaz de detectar; y que sólo hasta los $11 \mathrm{~Hz}$ se obtiene un valor de caudal representativo, medible por el transmisor. 
Por encontrarse en medio de la zona con comportamiento aproximadamente lineal, se aísla la respuesta transitoria para el escalón aplicado entre $25 \mathrm{~Hz} \mathrm{y} 30 \mathrm{~Hz}$, con el fin de obtener el modelo matemático lineal requerido a partir de estos datos experimentales. En la Fig. 9, la línea discontinua corresponde a la respuesta transitoria del caudal para esta señal tipo escalón de 5 $\mathrm{Hz}$, representada en la misma Figura. Ambas señales han sido referenciadas a cero para un tratamiento más cómodo de su información. Al ver esta gráfica, se hace evidente un retardo en la respuesta del sistema, cuyas principales razones son las rampas de aceleración y desaceleración seguidas por el variador de frecuencia frente a una consigna de velocidad específica, y la cabeza de presión que debe vencer la bomba, mencionada anteriormente.

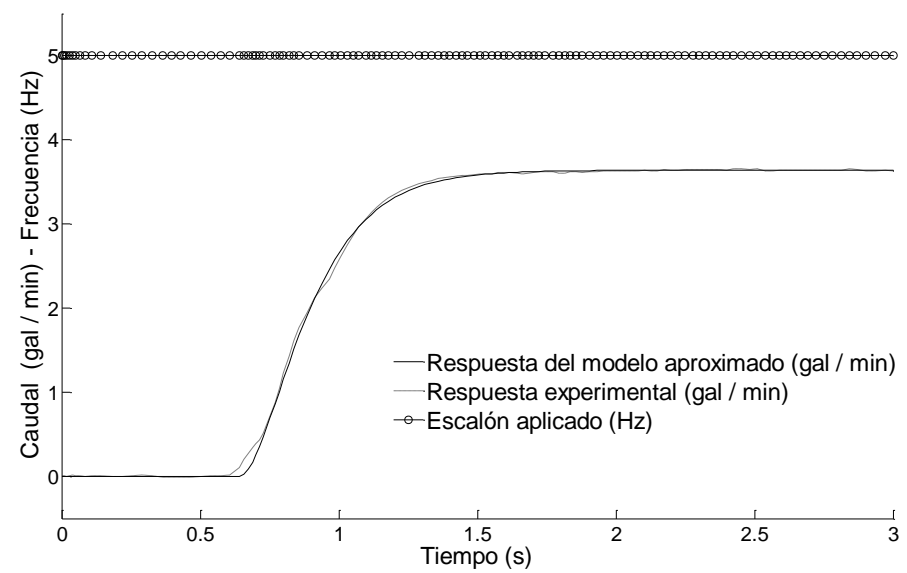

Fig. 9. Respuesta transitoria del sistema en la zona lineal y validación del modelo matemático calculado

Utilizando un aplicativo para la identificación de sistemas y considerando los datos experimentales, se pudo definir un modelo matemático lineal de tercer orden, cuya respuesta transitoria frente al mismo escalón de $5 \mathrm{~Hz}$ se muestra en la misma Fig. 9, identificada con la línea continua. La respuesta de este modelo entrega una correspondencia de $97,10 \%$ con los datos experimentales. Considerando que el retardo de tiempo 
correspondiente a este modelo es de 0.632 segundos y que el periodo de muestreo utilizado para estas señales fue de $1 \mathrm{~ms}$, la representación en espacio de estados del sistema según (1), está dada por:

$$
\begin{array}{cl}
A=\left[\begin{array}{ccc}
2.896 & 1.397 & 0.447 \\
2.0 & 0.0 & 0.0 \\
0.0 & 1.0 & 0.0
\end{array}\right] & B=\left[\begin{array}{c}
0.001953 \\
0.0 \\
0.0
\end{array}\right] \\
C_{m}=\left[\begin{array}{llll}
0.0003021 & 0.0005881 & 0.0001431
\end{array}\right] & D_{v m}=[0.0]
\end{array}
$$

No se incluyeron las demás matrices del modelo, dado que no se consideraron las perturbaciones que podrían afectar el comportamiento del sistema.

\subsection{Resultados Obtenidos}

Además del modelo matemático lineal de tercer orden con retardo calculado, se integraron las siguientes restricciones de operación en la formulación del problema de optimización:

Caudal mínimo: $y_{\min }=0 \mathrm{gal} / \mathrm{min}$

Caudal máximo: $y_{\max }=39 \mathrm{gal} / \mathrm{min}$

Frecuencia mínima: $u_{\min }=0 \mathrm{~Hz}$

Frecuencia máxima: $u_{\max }=60 \mathrm{~Hz}$

Variación de la frecuencia máxima: $\Delta u_{\max }=60 \mathrm{~Hz} / \mathrm{seg}$

Dado que no existen criterios claros para definir los otros parámetros requeridos por el controlador, éstos se seleccionaron haciendo diversas pruebas experimentales con el algoritmo. Finalmente, se utilizaron los siguientes datos:

Intervalo de control: 0,1 segundos

Horizonte de predicción: $n_{y}=10$ intervalos

Horizonte de control: $n_{u}=2$ intervalos

De estos parámetros, el Intervalo de Control es el que más influye sobre el desempeño del controlador: para valores mucho 
más pequeños que el indicado, el controlador no alcanza a procesar el algoritmo de optimización entregándose así una señal de control nula; si por el contrario se programa un Intervalo de Control más grande, se obtiene una regulación bastante pobre, ya que en este caso el algoritmo no puede responder a la velocidad de respuesta del sistema. Cabe resaltar en este punto que los valores iniciales que requieren los pesos del problema de automatización también exigen de un ajuste que puede afectar el desempeño del controlador.

La Fig. 10 muestra los resultados experimentales del control de caudal usando el CPM descrito. La línea discontinua corresponde a una señal de referencia de prueba arbitraria que cubre todo el rango de operación de la variable controlada, con escalones que varían entre $5 \mathrm{gal} / \mathrm{min}$ y $35 \mathrm{gal} / \mathrm{min}$, en forma ascendente y descendente. Mientras que la señal con línea continua corresponde al comportamiento real del caudal aplicando el CPM.

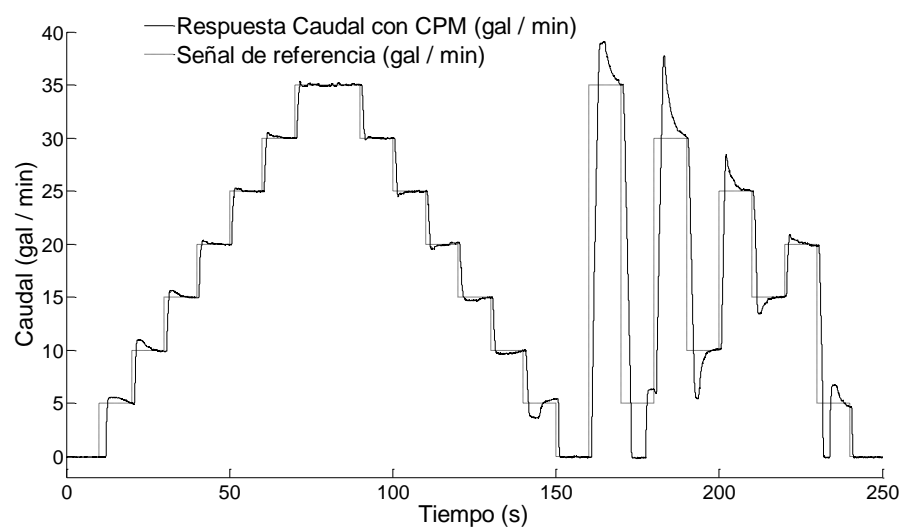

Fig. 10. Respuesta del sistema usando el CPM

En la Fig. 10 se observa que el CPM cumple satisfactoriamente con la regulación del caudal en la zona de operación con comportamiento aproximadamente lineal. Si bien es cierto que en la zona identificada como lineal, es decir, para caudales inferiores a $10 \mathrm{gal} / \mathrm{min}$, el controlador pierde el seguimiento de la señal de 
referencia, también es evidente su tendencia a recuperarla rápidamente en pocos segundos.

\subsection{Medidas de Desempeño}

Comparando el comportamiento de la señal de caudal frente a los escalones ascendentes y descendente, se aprecia que el CPM no tiene inconvenientes con el pequeño fenómeno de histéresis de la planta identificado en la curva de linealidad, que en el caso de un controlador PID, y sumado al componente no lineal de la planta, podría producir un comportamiento bastante inestable sobre la variable controlada, tal como se evidencia en la Fig. 11, donde se utilizó un controlador PID en el sistema, usando la misma señal de referencia, que presentó un comportamiento aceptable en la regulación de caudal inicialmente, pero que progresivamente fue perdiendo su capacidad de respuesta por los fenómenos mencionados.

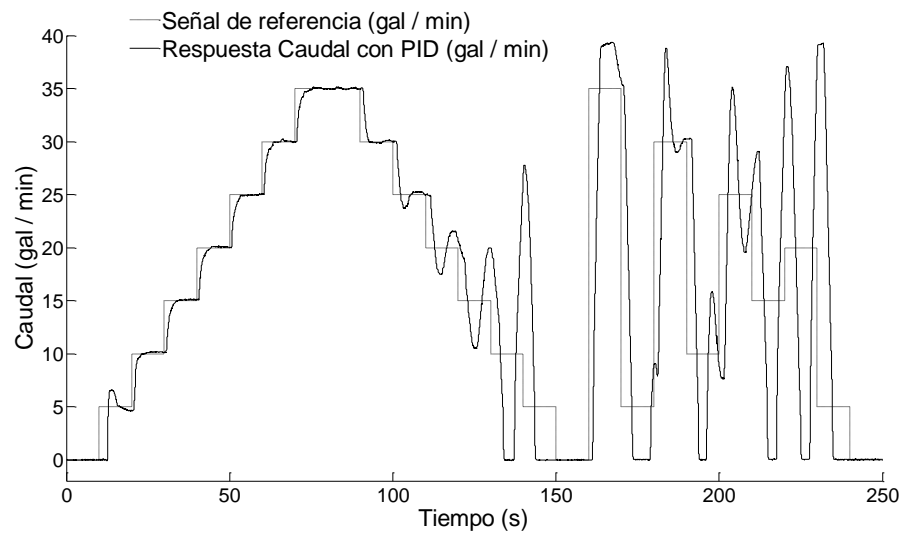

Fig. 11. Respuesta del sistema usando un controlador PID

Con el CPM se evidencia un muy buen comportamiento del controlador frente al retardo de tiempo que presenta la planta, lo que normalmente es difícil de enfrentar con otro tipo de reguladores. 


\section{CONCLUSIONES}

El CPM es una es una de las mejores alternativas existentes para reemplazar la regulación de variables físicas en los procesos industriales que no operan satisfactoriamente con los tradicionales controladores PID.

Aunque en el pasado la aplicación de esta tecnología ha sido exclusiva de un sector industrial particular, por su alto costo y complejidad en la implementación, se vislumbran grandes posibilidades en su aplicación práctica para todo tipo de sistemas que involucren la regulación de una o varias variables. Aún falta que el sector productivo en general reconozca las ventajas de este algoritmo y se preocupe por su expansión y popularización.

El CPM es un algoritmo de regulación muy versátil que presenta en la actualidad muchas características susceptibles de mejorar, por lo que puede promover una gran cantidad de investigaciones en el sector académico y los centros de investigación aplicada de las universidades y de la industria. El CPM es una tecnología que todavía está en desarrollo y que puede integrarse con muchos campos del saber de la ingeniería y la matemática.

No solamente un buen modelo matemático de la planta es suficiente para la implementación del algoritmo, en la práctica existen factores importantes que requieren que quien lo ponga en operación conozca con detalle el comportamiento de la planta y la estructura del algoritmo, lo que evidencia la necesidad de favorecer su acercamiento a los usuarios del controlador con sus principios de operación. En la práctica, se terminan haciendo ajustes de parámetros de forma similar a lo que sucede cuando se pone en operación un controlador PID; aunque en este caso con un grado de complejidad mayor.

Uno de los aportes más significativos que deja este trabajo de investigación, consistió en demostrar experimentalmente que con equipos de control e instrumentación industrial convencionales es posible lograr una implementación efectiva de un CPM y obtener de una forma sistemática un mejor desempeño en la regulación de variables de proceso como lo puede ser el caudal. 


\section{REFERENCIAS}

Bemporad, A., Morari, M. \& Lawrence, N., (2008); Model Predictive Control Toolbox, versión 2, 26-44, MathWorks, Massachusetts, United States.

Cao, Y. \& Lin, Z., (2005); Min-max MPC algorithm for LPV system subject to input saturation. IEE Proceedings. Control Theory \& Applications, 152(3), 266-272.

Fernández, E. \& Bordons, C., (2004a); Control predictivo: pasado, presente y futuro, Revista Iberoamericana de Automática e Informática Industrial, 1(3), 5-28.

Fernández, E. \& Bordons, C., (2004b); Model Predictive Control, 2a edición, 3-29, Springer-Verlag, London, United Kingdom.

Hirano, K., Yongsu, U. \& Kano, Y., (2001); A simulation of MPC application for linear motor position control, International Journal of Applied Electromagnetics \& Mechanics, 1(13), 181-185.

Jalili, M.\& Araabi, B., (2004); Neural Network based Predictive Control of a Heat Exchanger Nonlinear Process, Journal Of Electrical \& Electronics Engineering, 4(2), 1219-1226.

Lawrynczuk, M., (2007); A family of Model Predictive Control algorithms with artificial neural networks, Int. J. Appl. Math. Comput. Sci., 17(2), 217-232.

Maciejowski, J. M., (2002); Predictive control with constraints, 145-165, Prentice Hall, London, United Kingdom.

O'dwyer, A., (2006); Handbook of PI and PID controller tuning rules, 1-2, 2a edición, Imperial College Press, London, United Kingdom.

Qin, S. J. \& Badgwell, T. A., (2003); A survey of industrial model predictive control technology, Control Engineering Practice, 1(11), 733-764.

Ramírez, J. \& Londoño, C., (2007); Rediseño e implementación de un prototipo para la medición y control de nivel y caudal de agua, Tecno Lógicas, 1(1), 171-185.

Rodríguez, M. A. \& León, J., (2002); Control Predictivo basado en Modelo de un horno industrial, Revista Colombiana de Física, 34(1), 245-249. 